Laser and photodiode environmental evaluation for the Versatile Link project

This content has been downloaded from IOPscience. Please scroll down to see the full text. 2013 JINST 8 C02053

(http://iopscience.iop.org/1748-0221/8/02/C02053)

View the table of contents for this issue, or go to the journal homepage for more

Download details:

IP Address: 137.138.125.163

This content was downloaded on 08/07/2014 at 08:41

Please note that terms and conditions apply. 
Topical Workshop on Electronics for Particle Physics 2012, 17-21 SEPTEMBER 2012, OXFORD, U.K.

\title{
Laser and photodiode environmental evaluation for the Versatile Link project
}

\author{
J. Troska, ${ }^{a, 1}$ S. Seif El Nasr-Storey, ${ }^{a}$ S. Détraz, ${ }^{a}$ L. Olanterä, ${ }^{a}$ P. Stejskal, ${ }^{b}$ \\ C. Sigaud, ${ }^{a}$ C. Soós ${ }^{a}$ and F. Vasey ${ }^{a}$ \\ ${ }^{a} C E R N$ \\ 1211 Geneva 23, Switzerland \\ ${ }^{b}$ Blackett Laboratory, Imperial College, \\ London SW7 2AZ, U.K. \\ E-mail: jan.troska@cern.ch
}

\begin{abstract}
We summarize the results obtained in a series of radiation tests of candidate laser and photodiode components for use in the Versatile Transceiver (VTRx), the front-end component of the Versatile Link. We have carried out radiation testing at a full spectrum of sources (neutrons, pions, gammas) and can now compare the results and show that the range of components that meet the radiation tolerance requirements is rather large. In addition, devices have been operated in a high magnetic field to qualify them for use in (HL-) LHC detectors.
\end{abstract}

KEYWORDS: Radiation damage to electronic components; Optical detector readout concepts; Radiation-hard electronics

\footnotetext{
${ }^{1}$ Corresponding author.
} 


\section{Contents}

1 Introduction 1

2 Environmental specifications 2

3 Magnetic field testing 3

3.1 Test Setup 3

3.2 Results 3

4 Radiation testing 4

4.1 Gamma irradiation 5

4.1.1 Test Setup 5

4.1.2 Results 5

$\begin{array}{lll}4.2 & \text { System impact } & 6\end{array}$

$\begin{array}{lll}\text { 4.2.1 Lasers } & 6\end{array}$

4.2.2 Photodiodes 1

5 Conclusions 9

\section{Introduction}

The Versatile Link project [1] aims to develop the optoelectronic components required to build a $5 \mathrm{~Gb} / \mathrm{s}$, bi-directional, radiation hard optical link that bridges the $50-150 \mathrm{~m}$ distance between the front-end of the upgraded detectors at the HL-LHC and the back-ends located in the shielded counting rooms. An overview of the Versatile Link system is shown in figure 1. The link will support optical systems operating at either $850 \mathrm{~nm}$ and $1310 \mathrm{~nm}$ wavelength over multimode and singlemode optical fibres respectively. The Versatile link project is closely linked to the Gigabit Transceiver (GBT) [2] chip-set design project which aims to develop the ASICs needed to drive the optical components qualified in the framework of the Versatile Link project. The feasibility phase of the project is currently being completed [3]. This phase will show that all the link components are available and can meet the specifications of the final application both individually and when assembled as a full system.

Due to the increased luminosity of the HL-LHC, the on-detector components will have to withstand significant radiation levels: $2 \times 10^{15}$ neutrons $/ \mathrm{cm}^{2}$ plus $1 \times 10^{15}$ hadrons $/ \mathrm{cm}^{2}$ fluence and $500 \mathrm{kGy}$ total dose for future tracking detectors at $20 \mathrm{~cm}$ radius from the beam. These levels will be achieved after the luminosity upgrade foreseen for the LHC after the third planned long shutdown (LS3) during which the tracking detectors of ATLAS and CMS are foreseen to be replaced. Several detector upgrades are foreseen already in the second long shutdown (LS2) and these will be the first to deploy Versatile Link optical systems. 


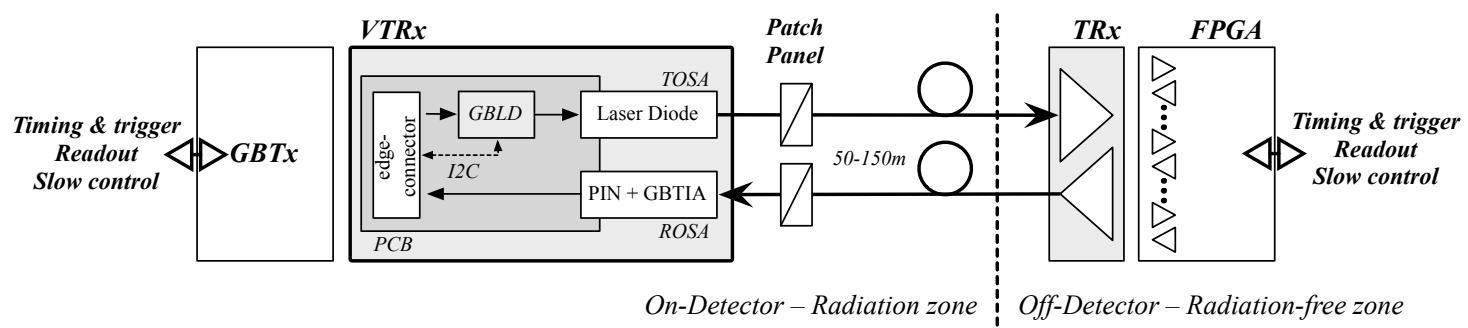

Figure 1. Overview of the Versatile Link system showing the components of the VTRx.

\section{Environmental specifications}

Detectors from all parts of the upgraded experiments are to be accommodated, which necessarily makes the environmental specifications more wide-ranging than they would be if only one specific detector were to be targeted. The environment of detectors at HL-LHC is characterised in terms of the radiation level, the temperature and the magnetic field present in the detector volume of interest.

The Versatile Link system specifications [4] define two levels of radiation tolerance. The first, Calorimeter grade, is targeted at radiation levels typical of those that will be experienced by HL-LHC calorimeter systems collecting $3000 \mathrm{fb}^{-1}$ of data up to the 2030 era. The second, Tracker grade, targets inner detectors that will operate over the same time-period of the HLLHC. The radiation tolerance levels are defined as follows: the Calorimeter grade must withstand $10 \mathrm{kGy}$ total dose and $5 \times 10^{14}$ neutrons $/ \mathrm{cm}^{2}$; while the Tracker grade must withstand $500 \mathrm{kGy}$ total dose, $2 \times 10^{15}$ neutrons $/ \mathrm{cm}^{2}$, and $1 \times 10^{15}$ hadrons $/ \mathrm{cm}^{2}$. The latter requirement is translated into a qualification level of $6 \times 10^{15} 20 \mathrm{MeV}$ neutrons $/ \mathrm{cm}^{2}$ using the neutron beam line at the Université Catholique de Louvain, Belgium (UCL). The effect of these levels of dose and fluence on the optoelectronics components needed to build the Versatile Link has been extensively investigated [5-8]. The last test to be carried out was a total dose test at a gamma source, the results of which are presented in section 4.1, below. The effect on the overall operation of the system will then be presented in section 4.2 .

The temperature environment ranges from the low temperatures required to operate silicon detectors successfully in radiation environments to the potentially slightly elevated temperatures of the rack environment of the back-end components, passing through the moderate temperatures typically found in the readout areas of the outer detectors of the HL-LHC experiments. The temperature specification for the Versatile link components has thus been set to $-30^{\circ} \mathrm{C}$ to $+60^{\circ} \mathrm{C}$ for the front-end components [4]. The impact of this temperature on the components and on link operation has been investigated and presented [9].

The magnetic field tolerance requirement is set by the CMS Magnet system that produces a $4 \mathrm{~T}$ field in the inner regions of CMS, the highest field level of all of the LHC experiments. The results of our study of the impact of magnetic fields on the versatile link components will be shown in section 3 . 
Table 1. Samples used for magnetic field test

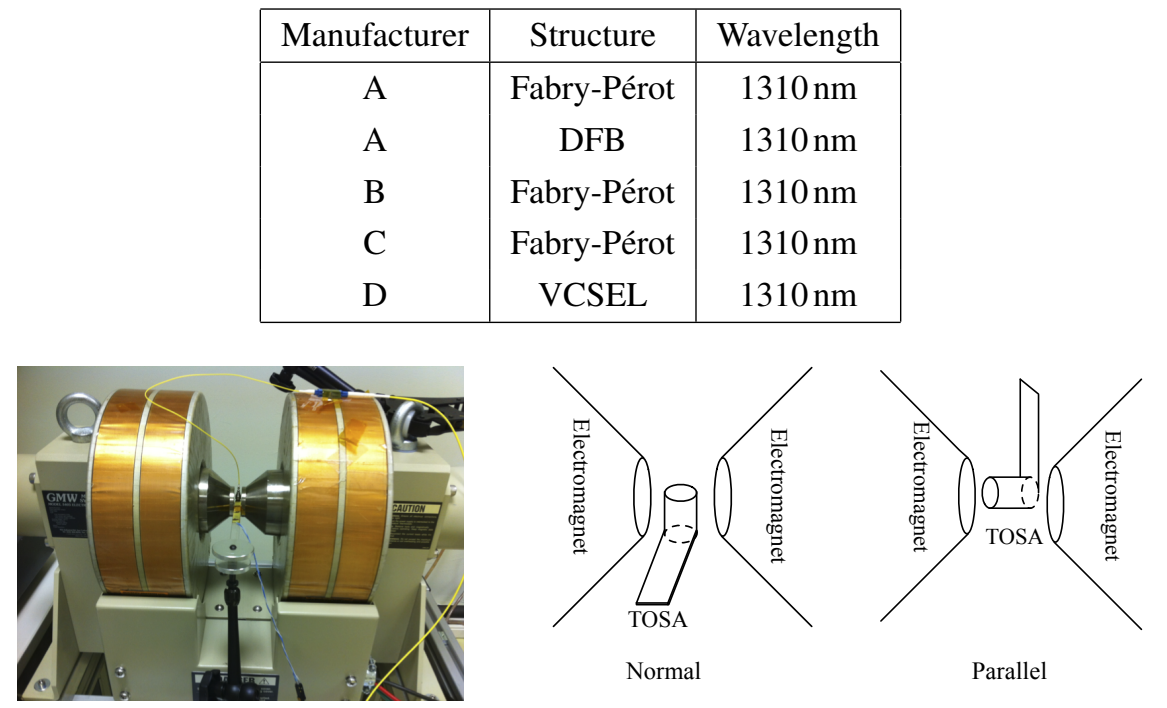

Figure 2. Setup used to apply the magnetic field to laser diodes.

\section{Magnetic field testing}

The behaviour of the components of the Versatile Link that will operate within the detector volumes must not deviate from the specifications even in the presence of a magnetic field. The devices that are potentially the most sensitive to the magnetic field are the laser diodes, that are packaged in so-called Transmitter Optical Sub-Assemblies (TOSA). The electromagnet in the CERN Quality Assurance and Reliability Testing (QART) lab was used to test the performance of the devices at different magnetic field strengths and orientations of the TOSAs with respect to the magnetic field.

\subsection{Test Setup}

A number of candidate devices were included in the test as shown in table 1. The devices under test (DUTs) were placed in the gap between the poles of the magnet (as shown in figure 2), and the strength of the magnetic field was varied by increasing the current supplied to the electromagnet. The magnet is capable of reaching a maximum field of $2.4 \mathrm{~T}$. The dimensions of the TOSAs used in this test placed a minimum limit on the size of the gap between the poles, and we were able to reach a field of $2.0 \mathrm{~T}$ when the TOSAs were placed perpendicular (see figure 2 ) to the magnetic field and $0.7 \mathrm{~T}$ when the TOSAs were placed parallel to the magnetic field. The output power, optical spectrum, and relative intensity noise (RIN) of each device were measured for each device for $0,0.5,1.5$, and $2.0 \mathrm{~T}$ with the TOSA normal to the magnetic field, and 0.0 and $0.7 \mathrm{~T}$ with the TOSA parallel to the magnetic field.

\subsection{Results}

The LI curves of the devices tested are shown in figure 3. A 60 percent decrease in the slope efficiency of the DFB laser was observed when it was placed in a $2 \mathrm{~T}$ field normal to the device; all other devices were unaffected by the magnetic field. The small difference visible for VCSEL(D) is 

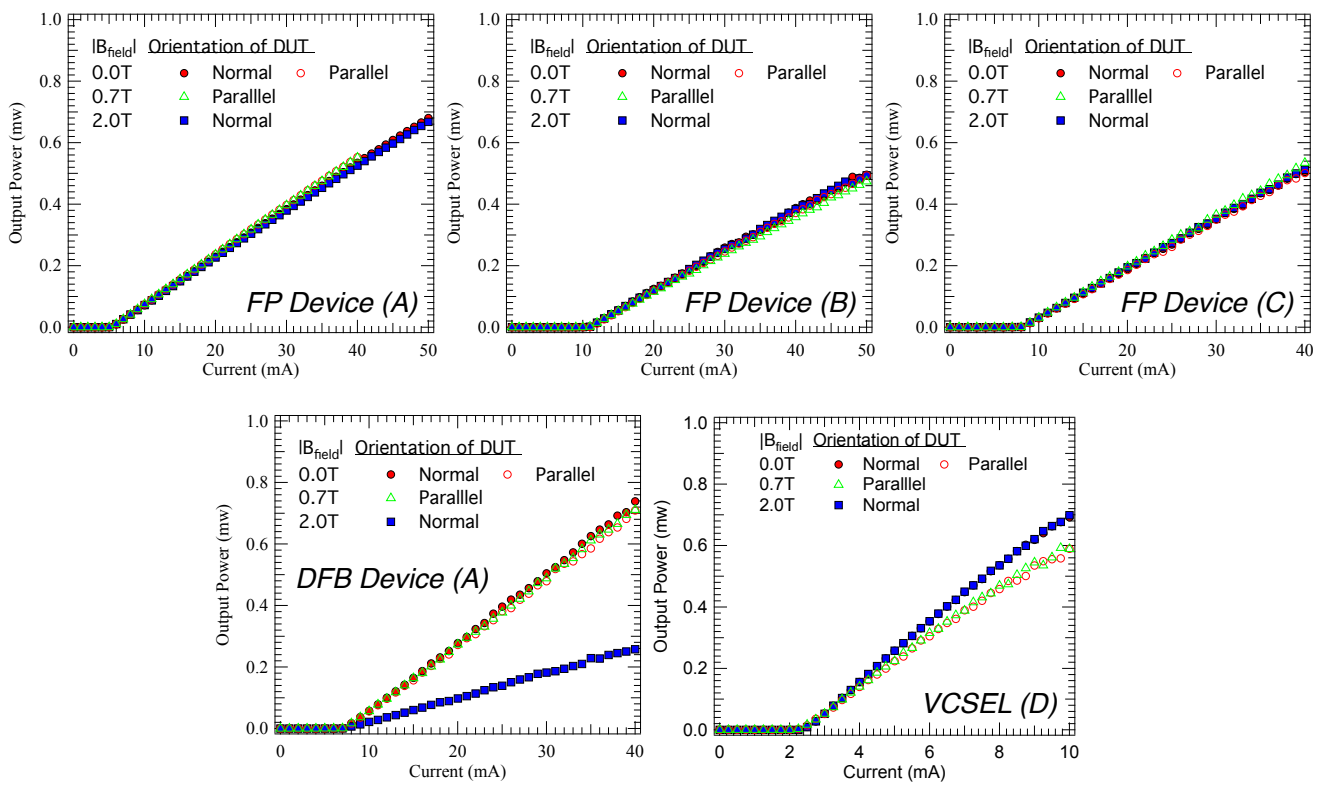

Figure 3. Impact of magnetic field on laser LI characteristics.

due to the difficulty in placing this TOSA in the magnet in the parallel orientation without bending the output fibre so much that the output power is slightly decreased. The same change is observed in the peak power of the optical spectra collected for the devices: only the spectrum of the DFB device was affected by the magnetic field, and in that case only the power at the central peak (and not it's position) was changed. DFB laser TOSAs always contain optical isolators to ensure their correct operation. These components are magnetic and were expected to be affected by the external field. DFB lasers are not candidate devices for use in the VTRx and were included as a control sample to make sure that we could observe something in case the other devices were insensitive to the applied magnetic field. The optical spectra of the other devices was unchanged with the magnetic field, as were the RIN spectra. These tests confirm that the candidate devices are insensitive to magnetic fields up to $2 \mathrm{~T}$ which is sufficient for early adopters of Versatile Link systems. For applications at the highest magnetic field of $4 \mathrm{~T}$ in the CMS Tracker may require further verification. The existing optical transmitters in the CMS Tracker systems - that are very similar laser structures to those tested here - were tested at higher magnetic fields [10] and found to be insensitive up to $10 \mathrm{~T}$. The qualification criterion for those same lasers was subsequently set to $2.5 \mathrm{~T}$ and thus we conclude that the results presented here would remain pertinent for the CMS Tracker application.

\section{Radiation testing}

Extensive radiation testing of lasers and photodiodes has been carried out in the framework of the Versatile Link project. Initial exploratory neutron irradiation of VCSELs and EELs carried out at the UCL cyclotron facility showed that $850 \mathrm{~nm}$ VCSELs are sufficiently rad-hard for Tracker applications as expected [5]. Long-wavelength VCSELs operating around $1300 \mathrm{~nm}$ or $1550 \mathrm{~nm}$, while they were found not to be as rad-hard as their $850 \mathrm{~nm}$ counterparts, could also be used. $1310 \mathrm{~nm}$ EELs were also found to be sufficiently rad-hard for Tracker applications, with one candi- 
date standing out as being significantly more resistant than others. In all these cases the increase in laser threshold current and attendant decrease in output efficiency remain within what can be compensated by the system. These findings were confirmed by an irradiation with $300 \mathrm{MeV} / \mathrm{c}$ pions at the Paul Scherrer Institute (Switzerland). The irradiation test with pions also showed that the pions of this energy - which dominate the radiation field in the final tracker applications - are typically twice as damaging as the neutrons used for earlier testing [5]. A model for laser damage has been developed that allows the prediction of damage at the lower fluxes that will be encountered in the final application [6]. Similar testing was carried out on photodiodes [5] which showed that their responsivity drops to zero after exposure to fluences of several $10^{15}$ neutrons $/ \mathrm{cm}^{2}$ and that this drop occurs at lower fluences and is faster for GaAs devices than for InGaAs ones. In addition, InGaAs photodiodes show increases in leakage current of many orders of magnitude while GaAs devices do not exhibit measurable increases. There is no appreciable annealing of the observed radiation damage. The responsivity drop implies the use of InGaAs photodiodes even for $850 \mathrm{~nm}$ applications where Tracker-grade radiation resistance is required, the radiation-induced penalty associated with the use of GaAs devices being simply too large. These findings were again confirmed in the pion irradiation and it was found that pions are approximately twice as damaging as the UCL neutrons. Single-event upset testing has shown that although particles traversing the receiving photodiodes can cause upsets, these upsets can be fully corrected by the error correction protocol built into the GBT system [7, 8].

The one outstanding test was the exposure to purely ionising radiation at a gamma source to confirm the expectation that the damage associated with the HL-LHC radiation environment is dominated by displacement damage due to particle irradiation. The results of this final test are presented below, followed by a discussion of the in-system effects of the damage due to particle irradiation.

\subsection{Gamma irradiation}

\subsubsection{Test Setup}

The gamma irradiation test was carried out at the IONISOS facility in Dagneux (France) at a dose rate of $1.2 \mathrm{kGy} / \mathrm{h}$. The samples given in table 2 were divided into two identical sets each containing two of each type of device. Sample set 1 was irradiated to $100 \mathrm{kGy}$ and set 2 to $1 \mathrm{MGy}$. The devices were shorted during the test to maximise the potential damage. In addition, a single device of each type was kept un-irradiated as a references.

The devices were tested before and after exposure. Each time a sample set was tested, the reference devices were also measured in order to gauge the measurement error associated with reproducing the same test result at test times separated by intervals of many weeks. LIV curves and optical output spectra were measured for lasers and the response curve was measured for photodiodes. Figure 4 shows some typical measurement curves with the extracted parameters.

\subsubsection{Results}

Figures 5 and 6 show that no significant damage was observed in any of the devices after exposure to gamma radiation, within the measurement uncertainty. Although some increase in leakage current was observed in the InGaAs devices, the level of increase is insignificant when compared 
Table 2. Samples used for gamma irradiation test, the labels indicate the manufacturer

\begin{tabular}{|c|c|c|c|}
\hline Laser & Structure & Wavelength & Type \\
\hline A & Fabry-Pérot & $1310 \mathrm{~nm}$ & Pin \\
A & VCSEL & $850 \mathrm{~nm}$ & Flex \\
B & Fabry-Pérot & $1310 \mathrm{~nm}$ & Pin \\
C & VCSEL & $850 \mathrm{~nm}$ & Flex \\
D & VCSEL & $1310 \mathrm{~nm}$ & Pin \\
\hline
\end{tabular}

\begin{tabular}{|c|c|c|}
\hline Photodiode & Material & Type \\
\hline E & InGaAs & Pin \\
E*(no lens) & InGaAs & Pin \\
E & GaAs & Pin \\
A & InGaAs & Flex \\
A & GaAs & Flex \\
\hline
\end{tabular}
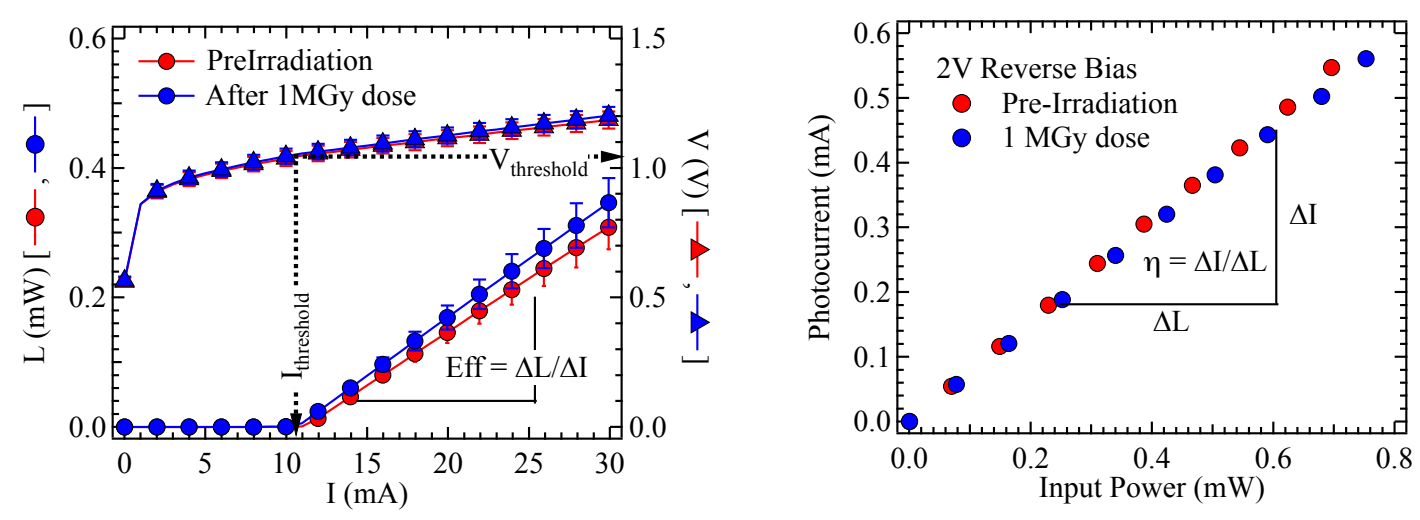

Figure 4. Typical LIV curves for lasers (left) and response curve for PINs (right) showing the measurements extracted before and after exposure to gammas.

to the many orders of magnitude increase typical of a particle irradiation to HL-LHC levels. The shaded boxes in figures 5 and 6 represent the measurement uncertainty that is defined as the spread in values measured on the reference devices, where the reference symbols represent the average of the measurements. The uncertainty is large because the measurements were carried out many weeks apart to allow such a high dose to be accumulated. The spectral measurements in particular are very sensitive to the temperature of the samples which was not controlled in the these tests.

\subsection{System impact}

\subsubsection{Lasers}

The concern at the system level is to be able to maintain a certain Optical Modulation Amplitude (OMA) during the operational lifetime of the lasers over which they are exposed to particle irradiation. The custom-designed GigaBit Laser Driver (GBLD) [11] includes two features to mitigate the effect of radiation: a programmable bias current to compensate for the increased laser threshold current; and a programmable modulation current to mitigate the decrease in laser slope efficiency. Figure 7 shows the effect of pion irradiation on the LIV characteristics of two VCSELs that are candidates for use in the VTRx. Figure 8 shows the change in bias current required to compensate the increase in threshold current as well as the upper limit on the bias current imposed by the compliance voltage limit of the GBLD power rail. The maximum bias current available from the GBLD is $45 \mathrm{~mA}$ to match the needs of edge-emitting lasers. $45 \mathrm{~mA}$ is clearly sufficient for these VCSEL candidates, where the expected shift is of the order of a few $\mathrm{mA}$. The fact that the solid and dashed 

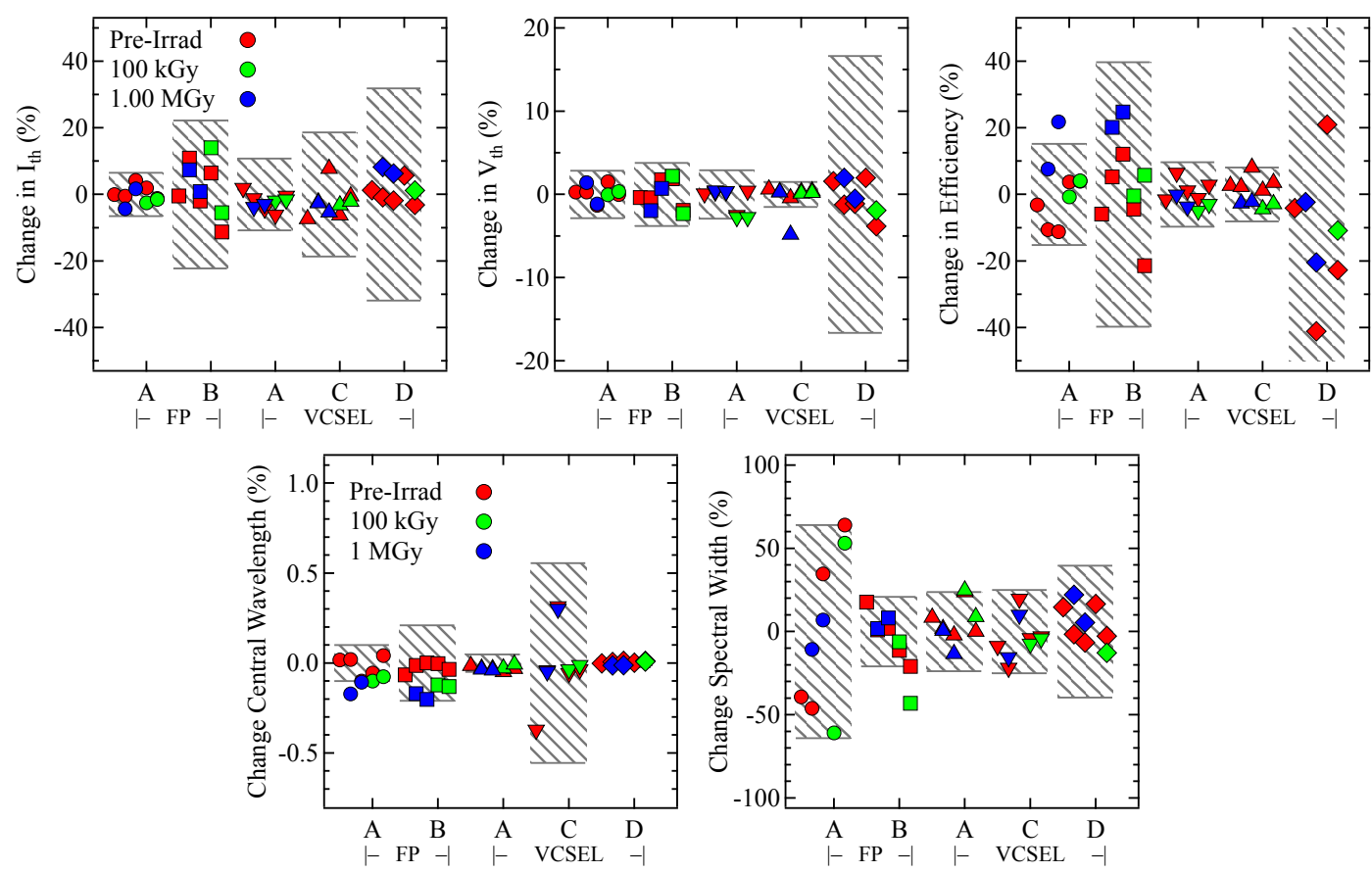

Figure 5. Changes observed in laser parameters after exposure to $100 \mathrm{kGy}$ and $1 \mathrm{MGy}$. A negative change would imply damage to the component due to the irradiation. The shaded regions represent the measurement uncertainty.
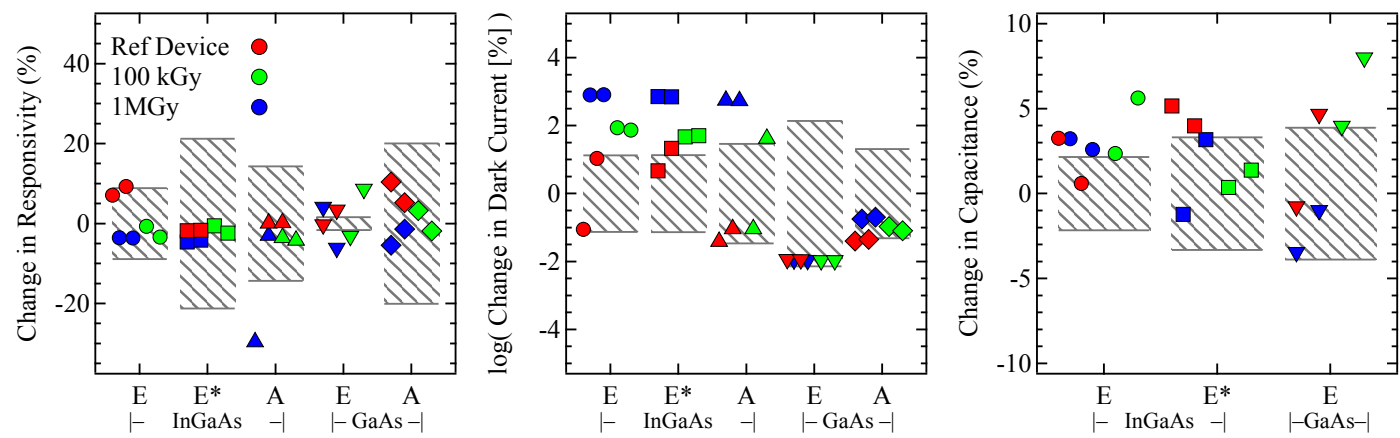

Figure 6. Changes observed in photodiode parameters after exposure to $100 \mathrm{kGy}$ and $1 \mathrm{MGy}$. The inset photograph shows dirt on the ROSA that lead to an artificially reduced responsivity after exposure. The increased leakage current in InGaAs devices is clearly visible. Shaded regions represent the measurement uncertainty.

lines of figure 8 do not intersect at the fluence level of interest shows that sufficient voltage headroom is also available for full mitigation of threshold current changes, although this is marginal in the case of VCSEL J. The right-hand plot of figure 8 shows that the slope efficiency may drop by 50 percent. The modulation current needed to reach the pre-irradiation minimum OMA specified for the VTRx is $5 \mathrm{~mA}$ for the minimum allowed laser slope efficiency. The maximum modulation current of the GBLD being $12 \mathrm{~mA}$ means that a 50 percent decrease in slope efficiency can be fully mitigated by increasing the modulation current. 

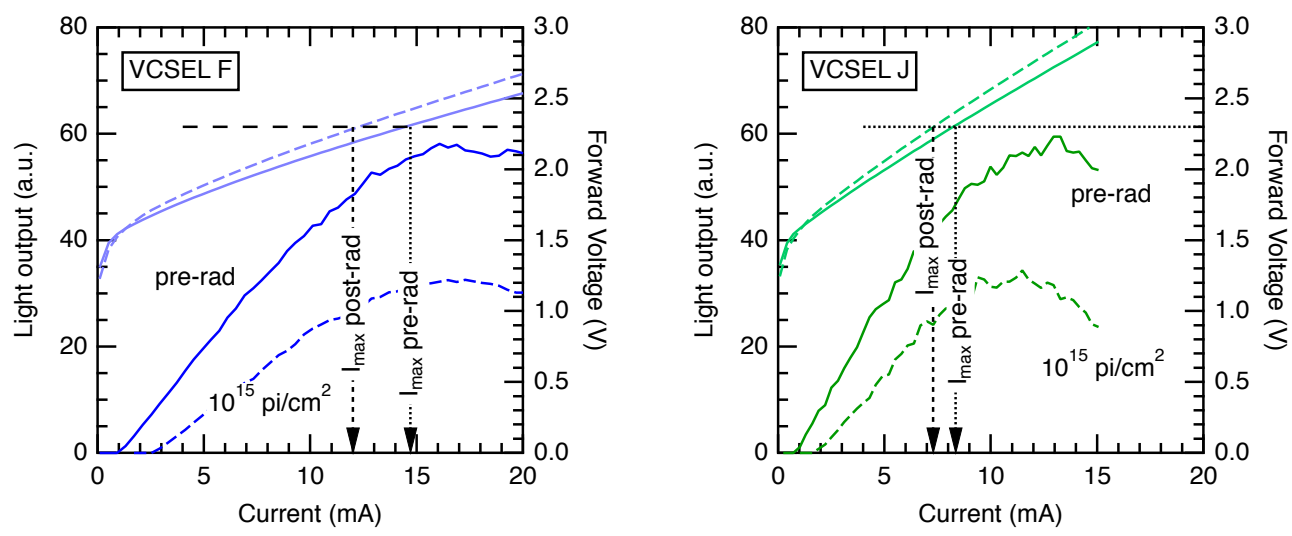

Figure 7. Impact of pion irradiation on the LIV curves of two VCSEL candidates.
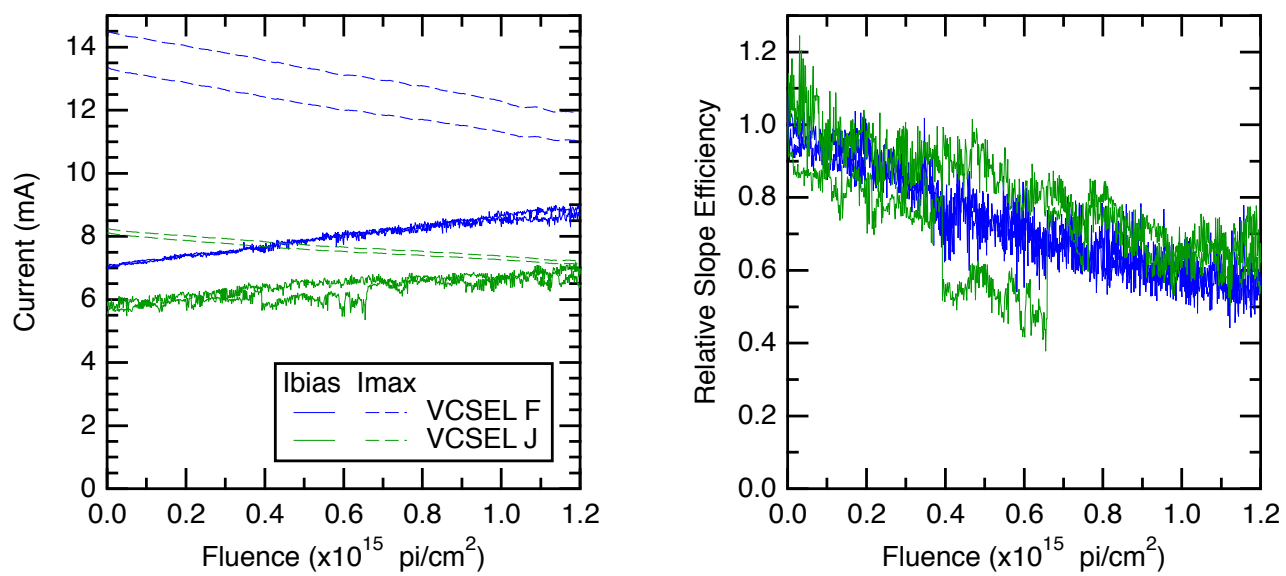

Figure 8. (Left) Impact of pion irradiation on the bias current needed by and maximum current available to two VCSEL candidates. (Right) Impact of pion irradiation on VCSEL slope efficiency.

\subsubsection{Photodiodes}

The degradation of the photodiode responsivity and leakage current must be taken into account in the calculation of the overall system optical power budget that is part of the system specifications. Figure 9 shows the measured and extrapolated penalty levels for InGaAs and GaAs photodiodes. InGaAs photodiodes with optimised anti-reflection coatings can achieve the same responsivity at $850 \mathrm{~nm}$ as their GaAs counterparts. The radiation-induced penalty of using GaAs photodiodes in a HL-LHC Tracker environment would be $9.6 \mathrm{~dB}$, to be compared to the $5.1 \mathrm{~dB}$ InGaAs penalty. We therefore propose to use InGaAs photodiodes also for the multimode Tracker systems operating at $850 \mathrm{~nm}$. The additional penalty due to the impact of the increased leakage current on the GBTIA is rather small at $0.3 \mathrm{~dB}$. This is a credit to the careful design of the GBTIA receiving amplifier that is able to minimise the impact of the leakage current increases in InGaAs photodiodes that can reach the level of $1 \mathrm{~mA}$ at Tracker radiation levels. 

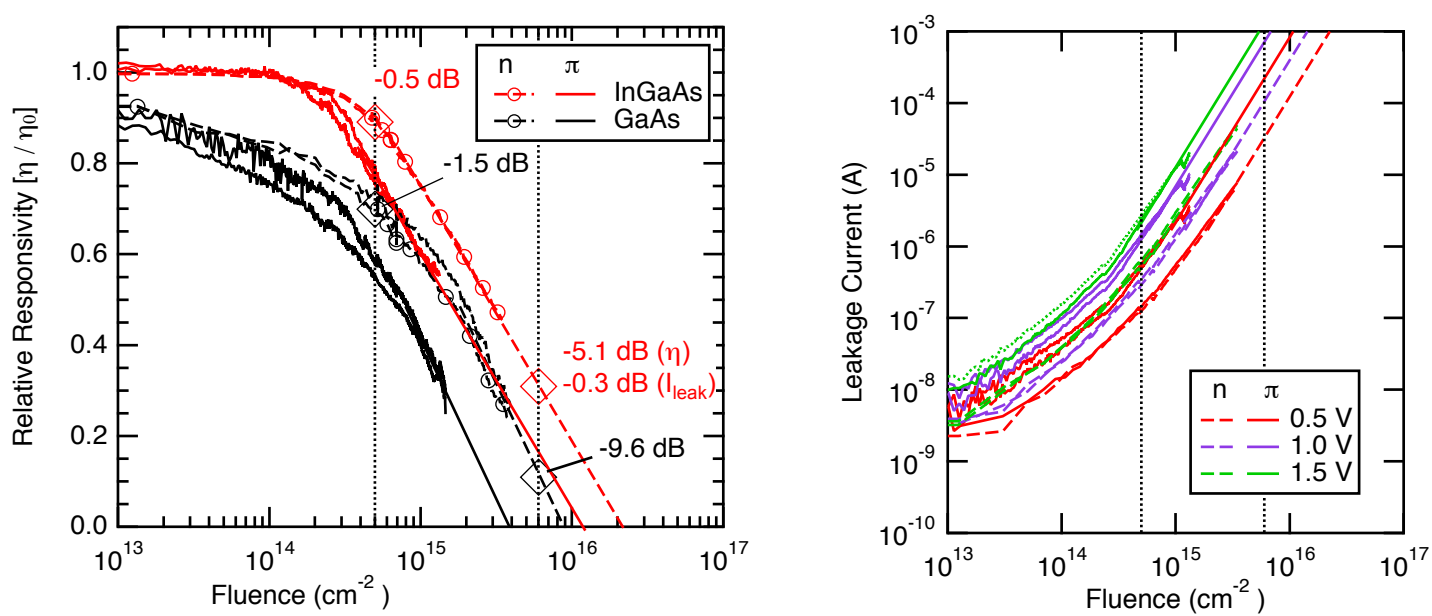

Figure 9. Drop in photodiode responsivity (left) and increase in leakage current (right) during particle irradiation. The two vertical dashed lines at $5 \times 10^{14} / \mathrm{cm}^{2}$ and $6 \times 10^{15} / \mathrm{cm}^{2}$ represent the qualification levels for Calorimeter and Tracker grade parts respectively.

\section{Conclusions}

A full picture of the radiation effects in candidate components now exists and shows that several suitable laser and photodiode devices are commercially available from different manufacturers. These candidates will operate in the low temperature, high magnetic field and high radiation environments of upgraded HL-LHC detectors. We have shown that the radiation effects can be mitigated with the programmable modulation and bias currents of the laser driver for the transmit side of the VTRx. On the receive side of the VTRx, the drop in responsivity can be compensated for at the system level by increasing the power in the off-detector transmitter; while the increased leakage current can be accommodated by a suitable design of receiving amplifier. These findings open the way to the formal qualification and procurement of devices for deployment in the upcoming LHC detector upgrades.

\section{Acknowledgments}

The authors would like to graciously acknowledge the support of the EU-funded 7th Framework Marie-Curie ACEOLE programme.

\section{References}

[1] CMS collaboration, L. Amaral et al., The versatile link, a common project for super-LHC, 2009 JINST 4 P12003.

[2] P. Moreira, A. Marchioro, and K. Kloukinas, The GBT: A proposed architecture for multi-Gb/s data transmission in high energy physics, in Proceedings of TWEPP 2007, the Topical Workshop on Electronics for Particle Physics, CERN-2007-007 (2007).

[3] F. Vasey et al., The Versatile Link common project: feasibility report, 2012 JINST 7 C01075. 
[4] A. Xiang, Versatile Link Specification, Part 1: System, https://edms.cern.ch/document/1140664/1 (2012).

[5] J. Troska et al., Radiation damage studies of lasers and photodiodes for use in Multi-Gb/s optical data links, IEEE Trans. Nucl. Sci. 58 (2011) 3103.

[6] P. Stejskal et al., Modelling radiation-effects and annealing in semiconductor lasers for use in future particle physics experiments, 2011 JINST 6 C12045.

[7] S. Seif El Nasr-Storey et al., Single-event upsets in photoreceivers for Multi-Gb/s SLHC data transmission, IEEE Trans. Nucl. Sci. 58 (2011) 3111.

[8] J. Troska et al., Single-Event Upset testing of the Versatile Transceiver, 2011 JINST 6 C11026.

[9] L. Olanterä et al., Temperature Characterization of Versatile Transceivers, in Proceedings of TWEPP 2012, Topical Workshop on Electronics for Particle Physics, 2012

[10] T. Bauer and M. Friedl, Performance of the CMS tracker analog optical link lasers exposed to high magnetic fields, CMS Internal Note 2003-030 (2003).

[11] G. Mazza et al., A $5 \mathrm{~Gb} / \mathrm{s}$ Radiation Tolerant Laser Driver in $0.13 \mu \mathrm{m}$ CMOS technology, in Proceedings of TWEPP 2009, the Topical Workshop on Electronics for Particle Physics, CERN-2009-006 (2009). 